\title{
Highly discordant T cell responses in individuals with recent exposure to household tuberculosis
}

\author{
A C Hesseling, ${ }^{1}$ A M Mandalakas, ${ }^{2} \mathrm{H}$ L Kirchner, ${ }^{3} \mathrm{~N} N$ Chegou, ${ }^{4}$ B J Marais, \\ $\mathrm{K}$ Stanley ${ }_{1}{ }^{4} \mathrm{X}$ Zhu, ${ }^{2} \mathrm{G}$ Black, ${ }^{4} \mathrm{~N}$ Beyers, ${ }^{1} \mathrm{G}$ Walz $\left.\right|^{4}$
}

${ }^{1}$ Desmond Tutu TB Centre, Department of Paediatrics and Child Health, Faculty of Health Sciences, Stellenbosch University, Tygerberg, South Africa; ${ }^{2}$ Department of Paediatrics, School of Medicine, Case Western Reserve University, Cleveland, Ohio, USA: ${ }^{3}$ Center for Health Research, Geisinger Health System, Danville, Pennsylvania, USA; ${ }^{4}$ DST/NRF Centre of Excellence in Biomedical

Tuberculosis Research and MRC Centre for Molecular and Cellular Biology, Division of Molecular Biology and Human Genetics, Faculty of Health Sciences, Stellenbosch University, Tygerberg, South Africa

Correspondence to:

Dr A C Hesseling, Desmond Tutu TB Centre, Department of Paediatrics and Child Health, Faculty of Health Sciences, Stellenbosch University, PO Box 19063, Tygerberg, 7505, South Africa; annekeh@sun.ac.za

Received 8 June 2007 Accepted 17 July 2008 Published Online First 5 August 2008

\section{ABSTRACT \\ Background: There are limited data comparing inter- feron- $\gamma$ release assays (IGRAs) for the detection of Mycobacterium tuberculosis infection in highly endemic settings.}

Methods: A cross-sectional household contact study was conducted to measure the agreement of two IGRAs in relation to the tuberculin skin test (TST) to detect $M$ tuberculosis infection and to assess the influence of $M$ tuberculosis exposure and age.

Results: In 82 individuals in household contact, 93\% of children and $42 \%$ of adults had a high $M$ tuberculosis contact score. The TST was positive in $78 \%$ of adults and $54 \%$ of children, the T-SPOT.TB was positive in $89 \%$ of children and $66 \%$ of adults and the QuantiFERON TB Gold (QTF) was positive in a similar proportion of adults and children (38.1\% and 39.6\%). In children there was poor agreement between the TST and T-SPOT.TB $(\kappa=-0.15)$ and the T-SPOT.TB and the OTF $(\kappa=-0.03)$, but good agreement between the TST and the QTF $(\kappa=0.78)$ using $10 \mathrm{~mm}$ cut-off. In adults there was fair to moderate agreement between the TST and T-SPOT.TB $(\kappa=0.38)$, the TST and OTF $(\kappa=0.34)$ and T-SPOT.TB and OTF $(\kappa=-0.50)$. High levels of exposure to $M$ tuberculosis were associated with at least a sevenfold odds of being T-SPOT.TB positive (95\% Cl 7.67 to 508.69) and a threefold odds of being OTF positive $(95 \% \mathrm{Cl} 3.02$ to 30.54). There was a significant difference in the magnitude of T-SPOT.TB early secretory antigenic target (ESAT)-6 and culture filtrate protein $10 \mathrm{kD}$ (CFP-10) spot counts between adults and children.

Conclusions: The T-SPOT.TB may be more sensitive than the TST or OTF for detecting recent $M$ tuberculosis infection in children. Differences between assays and the predictive utility of these findings for subsequent disease development should be prospectively assessed.

Every year almost nine million people contract tuberculosis and two million people die from the disease. ${ }^{1}$ The risk of progressing to active tuberculosis following Mycobacterium tuberculosis infection is high in young children during the first year following infection, with children aged $<1$ year of age having a $50-60 \%$ risk of disease progression. ${ }^{2} 3$ HIV-infected adults and children are also at high risk of disease progression and reactivation. ${ }^{4}$ Since up to $60 \%$ of young children diagnosed with active tuberculosis have a reported household contact with an infectious index case in high-burden settings, ${ }^{5}$ contact tracing of children and other individuals exposed to a household index case represents an opportunity for intensified tuberculosis control. ${ }^{6}$
The diagnosis of $M$ tuberculosis infection is complicated by the lack of a practical gold standard. Long-term follow-up of individuals to establish disease progression is therefore required to assess the predictive utility of diagnostic tools for tuberculosis. Although regarded as relatively robust for the diagnosis of $M$ tuberculosis infection, the tuberculin skin test (TST) has reduced specificity in populations with exposure to nontuberculous mycobacteria (NTM) and/or $M$ bovis Bacille Calmette-Guérin (BCG) vaccination. ${ }^{7}$ TST values of $\geqslant 10 \mathrm{~mm}$ are often regarded as positive in populations with normal immunity and who are BCG-vaccinated. ${ }^{9}$ TST sensitivity is limited in immune compromised individuals, very young and/or severely malnourished children or individuals with severe tuberculosis. ${ }^{7}$ The ability of the TST to detect recent $M$ tuberculosis infection may be limited, as demonstrated by a high proportion of individuals who convert their TST 6-12 weeks after documented $M$ tuberculosis exposure. ${ }^{10}$ Similarly, the proportion of individuals with $\mathrm{T}$ cell-based interferon gamma (IFN $\gamma$ ) release assay (IGRA) conversion following initial assessment may be as high as $24.6 \%$ at 3 months following negative initial responses. ${ }^{11}$ The potential benefits of an accurate diagnostic test of $M$ tuberculosis infection include timely institution of preventive or curative treatment, with reduction of both underdiagnosis and overdiagnosis and burdening of healthcare systems.

IGRAs using early secretory antigenic target (ESAT)- 6 and culture filtrate protein $10 \mathrm{kD}$ (CFP10) offer enhanced sensitivity and specificity by measuring IFN $\gamma$ production in response to antigens more specific to $M$ tuberculosis, and are not influenced by cross-reactivity to $M$ bovis BCG and most NTM. ${ }^{7}$ However, there are limited data on the utility of IGRAs to diagnose $M$ tuberculosis infection in persons from highly tuberculosis endemic settings. The two commercially available IGRAs include the ELISPOT-based T-SPOT.TB (Oxford Immunotec, UK) and the ELISA-based QuantiFERON TB Gold (Cellestis, Australia), with the in-tube form as the most recently available version, including TB 7.7 as an additional combined antigen. In the absence of a practical gold standard for $M$ tuberculosis infection, exposure to an infectious tuberculosis index case has been used as a surrogate measure of infection and has been shown to correlate well with ELISPOT IFN $\gamma$ responses in household contact and outbreak investigations. ${ }^{12-14}$ Direct comparisons of discordance between the two commercial IGRAs and the TST are limited, and there are few data on the 
comparison of both assays in relation to $M$ tuberculosis exposure as a surrogate measure of infection and the influence of age.

We conducted a cross-sectional household contact study to assess the agreement of two commercially available IGRAs in relation to the TST and investigated the impact of $M$ tuberculosis exposure and age on TST and IGRA responses to detect $M$ tuberculosis infection.

\section{METHODS}

\section{Study setting}

Our study was conducted in the Ravensmead/Uitsig (R/U) community in the Tygerberg District, Western Cape Province, South Africa, where the incidence of culture-confirmed tuberculosis in children aged $\leqslant 13$ years was 407/100 000 in 2004. ${ }^{15}$ The total tuberculosis notification rate was 841 per 100000 in $2002,{ }^{16}$ and the prevalence of HIV among patients with tuberculosis was 8\% in 2005 (Cape Town City Health). R/U is a well-characterised poor urban community of predominantly mixed ethnicity, with both formal and informal housing structures, often on the same residential property. Multiple families therefore often share the same residential address. There is a high level of ongoing $M$ tuberculosis transmission at the household and community level. ${ }^{17}$ In 2005 the calculated annual risk of $M$ tuberculosis infection in $\mathrm{R} / \mathrm{U}$ assessed through community-based school surveys was $3.5 \%$ among children aged 6-14 years, with $34.4 \%$ of children having a positive TST ( $\geqslant 10 \mathrm{~mm}$ ) (unpublished findings). BCG vaccine (Danish strain; 1331, Statens Serum Institute (SSI), Copenhagen, Denmark) is routinely given at birth.

\section{Participants}

From September 2005 through March 2006, HIV-uninfected children aged $0-5$ years and adults aged $\geqslant 15$ years in recent household contact with a tuberculosis index case were recruited. Individuals were eligible if exposed to a pulmonary tuberculosis index case and excluded if they were acutely ill, diagnosed with HIV infection, had active tuberculosis or were receiving isoniazid or antituberculosis treatment. Women were excluded if they were pregnant. All children with a household index case were referred for isoniazid preventive therapy in accordance with South African National Tuberculosis Control Program guidelines; all tuberculosis suspects were referred for further evaluation.

\section{Measures}

Data were collected on age, gender, BCG vaccination status (scar or documentation of vaccination record) and $M$ tuberculosis exposure. A household tuberculosis index case was defined as a person aged $\geqslant 15$ years with bacteriologically positive tuberculosis (sputum smear and/or culture positive) diagnosed at the $\mathrm{R} /$ $\mathrm{U}$ clinic during the previous 3 months and currently living in the same or neighbouring physical dwelling located on the same residential property. Caregivers and adult participants were interviewed to determine the participant's extent of contact with the index case during a typical week. A previously published $M$ tuberculosis contact score ${ }^{18}$ was modified for use in this study setting. The modified $M$ tuberculosis contact score was based on the assumptions that the gradient of $M$ tuberculosis exposure was a composite function of the infectivity of the index case, the duration of exposure and the proximity of exposure (table 1). The mean contact score was calculated for each participant. After exploration of cut-off values, the score was dichotomised as low $(<4)$ vs high exposure $(\geqslant 4)$ based on the score distribution in the sample.

Following phlebotomy (10 $\mathrm{ml}$ venous blood), a TST using 2 TU PPD RT 23 (Mantoux PPD, SSI) was placed on the volar aspect of the left forearm and read within 48-72 $\mathrm{h}$ using the ball-point pen and ruler method. TST reading by the study nurse was standardised through a large-scale community-based TST survey. A positive TST was defined as an induration of $\geqslant 10 \mathrm{~mm}$, in accordance with national guidelines.

Blood was transported at room temperature and processed within $3 \mathrm{~h}$ at the research laboratory. Laboratory analyses were blinded. Phlebotomy resulting in inadequate blood volumes, specimens not transported within the specified window period or clotted blood specimens were documented as "failed phlebotomy". If blood volumes were inadequate, the $\mathrm{T}$ SPOT.TB was preferentially completed.

The T-SPOT.TB and QuantiFERON TB Gold (OTF) tests were performed according to the manufacturers' instructions using positive and negative controls. All T-SPOT.TB tests were read by the same laboratory technician using an automated spot counter (Zeiss, Germany). The manufacturers' standard guidelines were used to define positive cut-off values for interpretation of IGRAs. HIV status was determined using a rapid screening test (AxSym, Abbott Diagnostics, Cape Town, South Africa) and confirmed by ELISA or by PCR in children aged $\leqslant 18$ months.

\section{Data analysis}

Data are expressed as frequencies and percentages for categorical variables and means for continuous variables. Agreement between tests was estimated using Cohen's kappa ( $\kappa)$ coefficient, while also describing the pattern of disagreement (discordant results). To aid in the interpretation of agreement and disagreement, estimates of the prevalence of positive results are presented. These estimates were compared between diagnostic tests using the McNemar $\chi^{2}$ test for correlated proportions. The association between each diagnostic test and the $M$ tuberculosis contact score (high vs low score) was estimated using logistic regression models, controlling for the potential confounding effect of age.

In addition to categorical representation of IGRAs based on manufacturers' guidelines, individual and total antigen responses for T-SPOT.TB (ESAT-6 and CFP-10, spot count per 250000 peripheral blood mononuclear cells) and IFN $\gamma$ OTF antigen response $(\mathrm{IU} / \mathrm{ml})$ were described as continuous measures. ${ }^{19}$ Comparisons between children and adults were performed using the Pearson $\chi^{2}$ and Wilcoxon rank sum tests for dichotomous and continuous tests results, respectively. Receiver operating characteristic (ROC) curves were generated to compare individual continuous IGRA antigen responses in relation to the $M$ tuberculosis contact score, which was used as a surrogate gold standard. Correlations were explored between T-SPOT.TB and OTF for the ESAT-6 and CFP-10 assay levels for children and adults using the Spearman rank correlation coefficient. The T-SPOT.TB spot count and QTF IFN $\gamma$ IU/ml were both transformed to the natural log scale for graphical purposes. Missing data and indeterminate results were excluded from analyses.

\section{RESULTS}

Eighty-two individuals were enrolled (29 children and 53 adults); 4 HIV-infected individuals (4.7\%) were excluded from the study. ${ }^{20}$ Two children with symptoms were referred for further investigation and excluded from the analysis based on 
Table 1 Mycobacterium tuberculosis contact score

\begin{tabular}{ll}
\hline Variable & $\begin{array}{l}\text { Weight } \\
\text { assigned }\end{array}$ \\
\hline Relationship to TB index case & \\
No known TB contact & 0 \\
Non-household TB contact & 1 \\
Relative/other contact in household with TB & 2 \\
Secondary caregiver (care provider during day) in & 3 \\
household with TB & \\
Primary caregiver in household with TB & 4 \\
Infectivity of TB index case & \\
No known TB contact & 0 \\
Sputum acid-fast negative & 2 \\
Sputum acid-fast positive & 4 \\
Type of exposure to TB index case & \\
No known exposure & 0 \\
Lives and sleeps in different house & 1 \\
Lives and sleeps in same house & 2 \\
Sleeps in same room & 3 \\
Duration (total hours) average contact per day with & \\
TB index case & 0 \\
No known contact & 1 \\
$0-3 \mathrm{~h}$ & 2 \\
4-7 h & 3 \\
8-11 h & 4 \\
Total contact score (maximum = 15) & \\
\hline TB tuberculosis &
\end{tabular}

TB, tuberculosis.

abnormal chest radiographs. The primary outcome measures and patient characteristics are summarised in table 2 . The mean $M$ tuberculosis contact score was significantly higher in children than in adults; $93 \%$ of children reported a high $M$ tuberculosis contact score $(\geqslant 4)$ compared with $42 \%$ of adults. In $55.2 \%$ of children the tuberculosis index case was the parent or primary caregiver.
Results were available for the TST and T-SPOT.TB for the entire sample. Owing to inadequate blood volumes, a smaller sample of 74 individuals had OTF responses. The TST distribution in millimetres is shown in fig 1; the TST was positive in $69 \%$ of individuals (median values in children vs adults in those with TST $>0 \mathrm{~mm}$ : $14.5 \mathrm{~mm}$ vs $19.0 \mathrm{~mm}$ ). There was no significant difference between the mean size of induration between BCG-vaccinated and unvaccinated subjects (21.43 $\mathrm{mm}$ vs $19.93 \mathrm{~mm}, \mathrm{p}=0.070$ ). None of the participants had a reported TST in the 6 months preceding enrolment. The TST was positive in significantly more adults than children $\left(78.0 \%\right.$ vs $\left.53.6 \%, \chi^{2}=5.0, p=0.02\right)$. Conversely, the $\mathrm{T}$ SPOT.TB was positive in more children than adults $(92.6 \%$ vs $\left.66.0 \%, \chi^{2}=6.7, p=0.01\right)$. OTF results were positive in adults and children $\left(39.6 \%\right.$ vs $\left.44.4 \%, \chi^{2}=0.13, p=0.72\right)$. The TSPOT.TB produced indeterminate results in $1 / 28$ children $(3.6 \%)$ and the QTF in $3 / 21$ children (14.3\%). No indeterminate results were observed in adults.

Discordant results between all three tests were common with percentage agreement ranging from $46.1 \%$ to $88.9 \%$ (table 3 ). Using different cut-off values for the TST did not improve the yield or test agreement significantly (proportion positive: $73.0 \%$, $69.2 \%$ and $65.4 \%$ for TST cut-off values of 5,10 and $15 \mathrm{~mm}$, respectively, in all subjects).

Table 4 shows the relation between exposure to $M$ tuberculosis and IGRA results. Participants with a high $M$ tuberculosis contact score were at least seven times more likely to be T-SPOT.TB positive than those with a low score (odds ratio (OR) 62.42, $95 \%$ CI 7.67 to 508.69 ) regardless of age, and at least three times more likely to be QTF positive (OR 9.60, 95\% CI 3.02 to 30.54 ). This relationship was confounded by age. The TST was not significantly associated with $M$ tuberculosis exposure in unadjusted or adjusted regression models. Using a higher $M$ tuberculosis exposure score cut-off value of 8 decreased the association between $M$ tuberculosis exposure and measures of infection (data not shown). All children were BCG-vaccinated

Table 2 Clinical characteristics and measures of Mycobacterium tuberculosis infection in adults and children in recent household contact with a tuberculosis index case

\begin{tabular}{|c|c|c|c|}
\hline & $\begin{array}{l}\text { All subjects } \\
(n=82)\end{array}$ & $\begin{array}{l}\text { Children } \\
(n=29)\end{array}$ & $\begin{array}{l}\text { Adults } \\
(n=53)\end{array}$ \\
\hline Mean (SD) age (years) $\dagger$ & $22.8(18.1)$ & $2.9(2.6)$ & $33.8(12.8)$ \\
\hline$\%$ with documented BCG vaccination & $75.6(62 / 82)$ & $100(29 / 29)$ & $62.2(33 / 53)$ \\
\hline \multicolumn{4}{|l|}{ TST $(\%) \dagger$} \\
\hline Positive* $\dagger$ & $69.2(54 / 78)$ & $53.6(15 / 28)$ & $78.0(39 / 50)$ \\
\hline Not read & $4.9(4 / 82)$ & $3.5(1 / 29)$ & $5.7(3 / 53)$ \\
\hline \multicolumn{4}{|l|}{ T-SPOT.TB $(\%)$} \\
\hline Positive $\uparrow$ & $75.0(60 / 80)$ & $92.6(25 / 27)$ & $66.0(35 / 53)$ \\
\hline Indeterminate & $1.2(1 / 81)$ & $3.6(1 / 28)$ & $0(0 / 53)$ \\
\hline \multicolumn{4}{|l|}{ QTF (\%) } \\
\hline Positive & $40.9(29 / 71)$ & $44.4(8 / 18)$ & $39.6(21 / 53)$ \\
\hline Indeterminate & $4.1(3 / 74)$ & $14.3(3 / 21)$ & $0(0 / 53)$ \\
\hline Mean (SD) $M$ tuberculosis contact score $\dagger$ & $6.4(5.6)$ & $10.2(3.5)$ & $4.3(5.4)$ \\
\hline $\begin{array}{l}\text { Proportion with high } M \text { tuberculosis contact } \\
\text { score }(\geqslant 4)+\dagger\end{array}$ & $59.8(49 / 82)$ & $93.1(27 / 29)$ & $41.5(22 / 53)$ \\
\hline \multicolumn{4}{|c|}{ 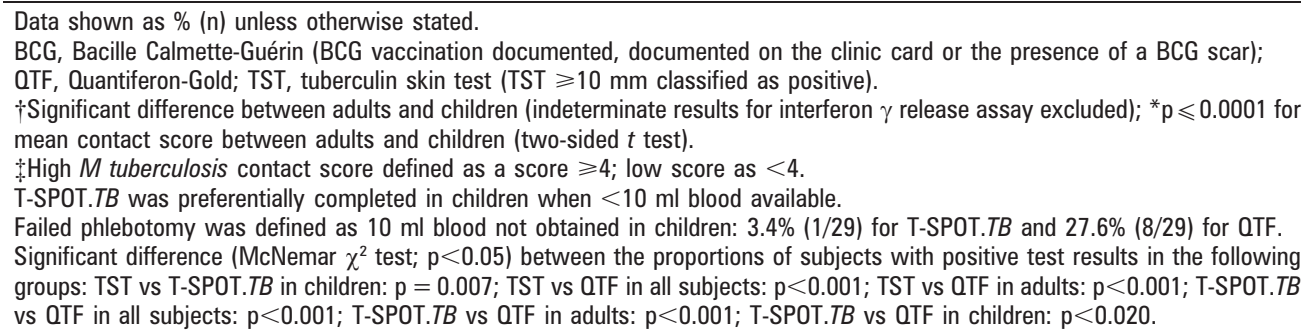 } \\
\hline
\end{tabular}




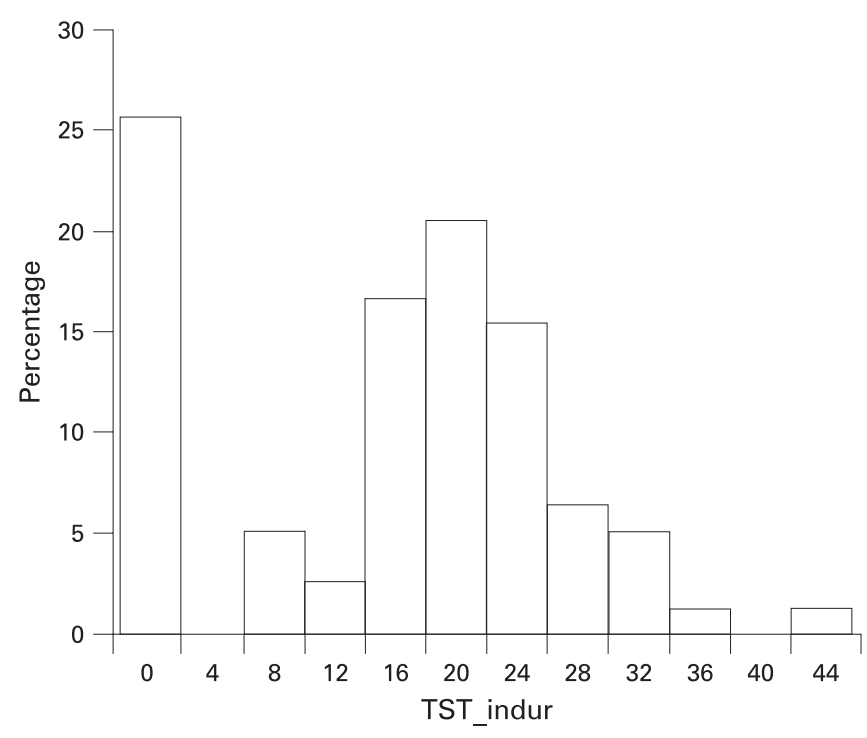

Figure 1 Mantoux tuberculin skin test distribution in HIV-uninfected adults and children in recent household contact with a tuberculosis index case. Mantoux tuberculin skin test (TST) induration in millimetres.

and had a BCG scar; in adults (data not shown), the presence of a BCG scar did not confound the relationship between exposure to $M$ tuberculosis and test results.

The mean (SD) T-SPOT.TB ESAT-6 response was higher than the CFP-10 response in all participants (111.7 (134.5) vs 26.6 (44.4); Wilcoxon sign test, $\mathrm{p} \leqslant 0.001)$. In contrast, the mean (SD) OTF CFP-10 response was higher than the mean ESAT-6 response in all participants (5.1 (10.0) vs 1.5 (3.5); Wilcoxon sign test, $p \leqslant 0.001)$. There was a significant difference in the magnitude of the T-SPOT.TB ESAT- 6 and CFP-10 spot counts between adults and children (mean (SD) ESAT-6 response in children 162.7 (144.0) vs 84.8 (122.2) in adults; Wilcoxon sign test, $p=0.002)$. In contrast, there was no difference between $T$ SPOT.TB CFP-10 responses in adults and children (mean (SD) response 29.9 (54.6) vs 24.9 (38.5), respectively). The magnitude of the QTF ESAT- 6 response was similar in children and adults (mean (SD) response 4.0 (4.6) vs 1.1 (3.0); $p=0.49$ ); the mean (SD) QTF CFP-10 response in children was 8.4 (14.4) compared with $3.8(7.6)$ in adults $(p=0.61)$. Exploration of correlation coefficients, although not significant, suggested an age-related correlation between QTF and T-SPOT.TB CFP-10 responses (adults $r=0.71$; children $r=0.39 ; p=0.10$ for comparison of coefficients between groups) and a lesser correlation between QTF and T-SPOT.TB ESAT-6 responses (adults $r=0.34$; children $r=-0.06 ; p=0.14$ for comparison of coefficients between groups; figs 2 and 3 ).

ROC curves describe the sensitivity and specificity of ESAT-6 and CFP-10 responses compared with high $M$ tuberculosis exposure ( $\geqslant 4$ ) (fig 4). T-SPOT.TB ESAT-6 responses appeared to offer the highest sensitivity and specificity of any single antigen (area under the curve (AUC) $=0.933$ ). T-SPOT.TB CFP10 individual responses were less sensitive and specific (AUC $=0.687$ ). The combined ESAT-6 CFP-10 T-SPOT.TB response appeared to be largely influenced by the ESAT-6 component. The sensitivity and specificity of the QTF ESAT-6 and CFP-10 responses (AUC) were 0.721 and 0.825 , respectively.

\section{DISCUSSION}

There was a high prevalence of $M$ tuberculosis infection in HIVuninfected children and adults in this study population with documented recent household exposure to tuberculosis. We found significant discordance between TST, T-SPOT.TB and QTF. Participants with a high degree of exposure to $M$ tuberculosis had a high risk of having a positive IGRA result, regardless of age. The high prevalence of infection is consistent with recently published data from adults in a neighbouring high incidence community in Cape Town where discordant results were also detected, ${ }^{21}$ although that study did not correlate test results with degree of $M$ tuberculosis exposure or age.

Agreement between the three tests in children varied dramatically. Widely discrepant results between QTF and TST in children have been reported in both low and high incidence settings. ${ }^{22-24}$ The T-SPOT.TB was positive in a significantly higher proportion of children than the TST and the QTF. These results suggest that the T-SPOT.TB may be a more sensitive measure of $M$ tuberculosis infection than the TST or QTF in children with recent exposure, and appeared to be largely driven by robust ESAT- 6 responses. The most pronounced disagreement between the results in children was between the $\mathrm{T}$ SPOT.TB and the TST or QTF, whereas the TST and the QTF had good agreement. Our data also suggest improved specificity of both IGRAs compared with the TST. This could be explained by the fact that TST responses in older individuals are continually boosted by a variety of recurrent exposures, including $\mathrm{NTM}^{25}$ and $M$ tuberculosis. The clinical importance of these observed discordances is unknown. Studies incorporating serial follow-up measures in different populations will support further exploration of these differences and the predictive utility of IGRAs to identify acute and latent infection and the subsequent risk of progressing to active tuberculosis.

Children had a higher mean $M$ tuberculosis contact score than adults, reflecting the importance of proximity of contact and

Table 3 Comparison of TST and IGRA responses in adults and children in recent household contact with a tuberculosis index case

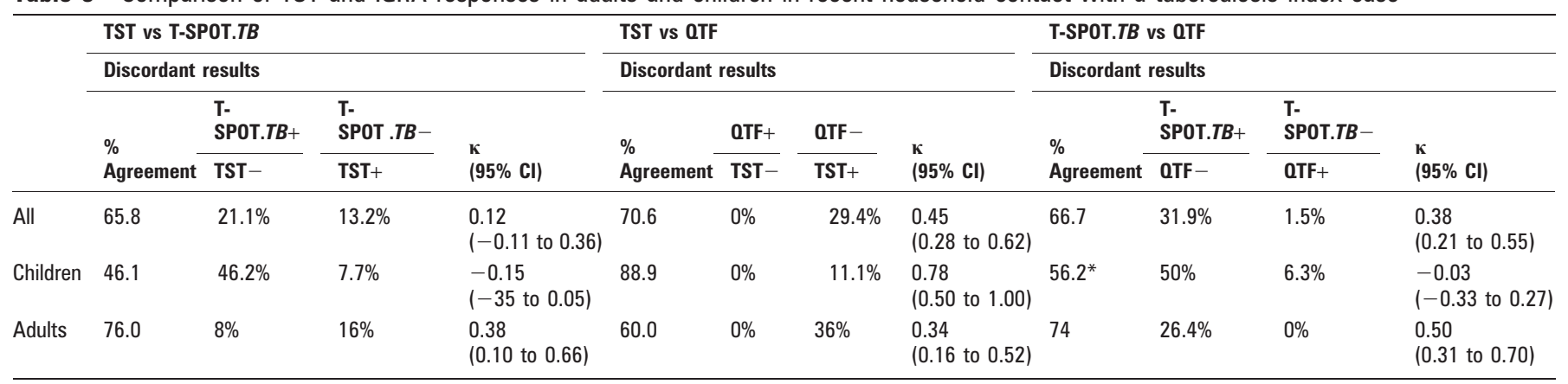


Table 4 Logistic regression of dichotomous measures of Mycobacterium tuberculosis infection in relation to exposure and age in adults and children in recent household contact with a tuberculosis index case

\begin{tabular}{|c|c|c|c|c|c|c|}
\hline \multirow[b]{4}{*}{ Covariates } & \multicolumn{6}{|c|}{ Measures of $M$ tuberculosis infection } \\
\hline & \multicolumn{2}{|l|}{ TST positive } & \multicolumn{2}{|l|}{ T SPOT.TB positive } & \multicolumn{2}{|l|}{ QTF positive } \\
\hline & Unadjusted & Age adjusted & Unadjusted & Age adjusted & Unadjusted & Age adjusted \\
\hline & OR $(95 \% \mathrm{CI})$ & OR (95\% Cl) & OR $(95 \% \mathrm{CI})$ & OR $(95 \% \mathrm{CI})$ & OR $(95 \%$ Cl) & OR (95\% Cl) \\
\hline $\begin{array}{l}\text { M tuberculosis } \\
\text { contact score } \geqslant 4\end{array}$ & $1.56(0.59$ to 4.15$)$ & $3.83(1.05$ to 14.03$)$ & 62.42 (7.67 to 508.69$)$ & $38.40(7.59$ to 616.11$)$ & $9.60(3.02$ to 30.54$)$ & 14.94 (4.02 to 55.58$)$ \\
\hline Age in years & - & $1.07(1.02$ to 1.11$)$ & - & $1.01(0.96$ to 1.06$)$ & - & 1.04 (1.01 to 1.08$)$ \\
\hline
\end{tabular}

$\mathrm{Cl}$, confidence interval; OR, odds ratio; OTF, Quantiferon-Gold; TST, tuberculin skin test (positive: $\geqslant 10 \mathrm{~mm}$ ).

the duration of exposure to $M$ tuberculosis experienced by young children. In the majority of cases the index case was the child's parent or primary caregiver, consistent with previous findings. ${ }^{26}$ A key consideration for the interpretation of our findings is that the majority of children had recent and significant documented household exposure, whereas many adults had less reported household exposure although they may have had previous or remote $M$ tuberculosis exposure. Adults had a higher probability of being TST positive (78\%) but a lower probability of being IGRA positive (66\% for T-SPOT.TB vs $39.6 \%$ for OTF), suggesting higher IGRA specificity but lower sensitivity in the presence of recent exposure.

Based on ROC analysis, T-SPOT.TB ESAT-6 responses were more strongly associated with recent $M$ tuberculosis exposure than CFP-10 responses. An interesting finding is that the mean T-SPOT.TB and OTF responses were different for ESAT-6 and CFP-10, with higher mean values observed for T-SPOT.TB ESAT-6 responses than for CFP-10 responses, but that higher OTF CFP-10 than ESAT-6 responses were observed. The ELISpot-based technique measures the number of IFN $\gamma$-producing cells while the ELISA technique (OTF) measures the accumulation of IFN $\gamma$ over time. Since not every IFN $\gamma$ producing cell produces identical amounts of IFN $\gamma$, a linear relationship between production and numbers of producing cells cannot be expected. Therefore, despite the association

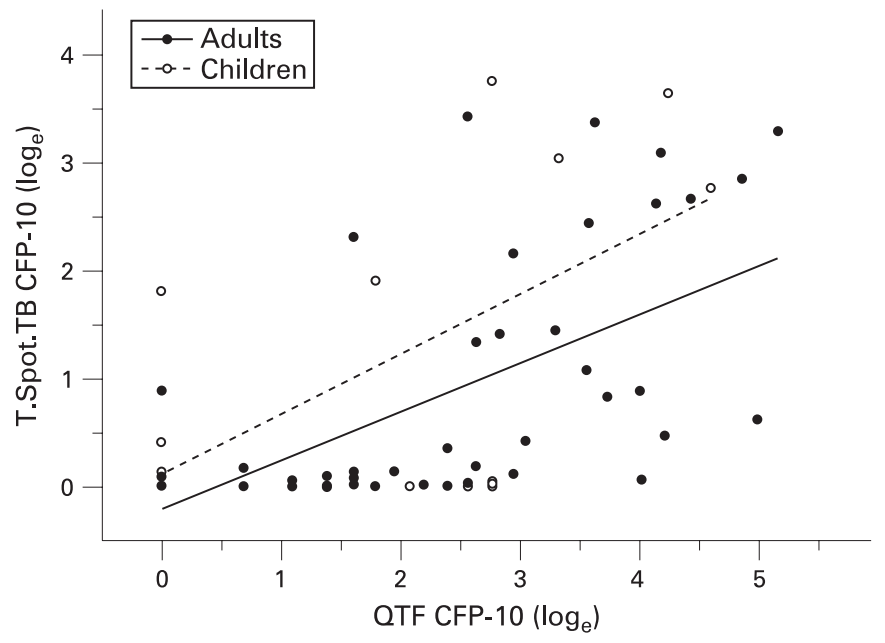

Figure 2 Correlation between interferon $\gamma($ IFN $\gamma)$ culture filtrate protein 10 kD (CFP-10) responses measured by QuantiFERON TB Gold (OTF) and T-SPOT.TB in adults and children. Vertical axis: T-SPOT.TB CFP-10 IFN $\gamma$ responses (spot count per 250000 peripheral blood mononuclear cells); horizontal axis: QTF IFN $\gamma$ CFP-10 response (IU/ml); both measures are reflected on the natural log scale. Spearman rank correlation coefficient for adults $r=0.71$ and for children $r=0.39 ; p=0.097$ for comparison of coefficients between groups. between the ELISA and ELISpot results, there is no absolute correlation, as demonstrated by our results and those of others. ${ }^{27}$ Leyten et al have proposed that the two tests appear to differ in their sensitivity for detecting cells producing low levels of IFN $\gamma$, which may partly explain these observed discrepancies. ${ }^{28}$ Bennekov et al demonstrated that, in an ELISA-based assay, most of the IFN $\gamma$ produced came from a subset of cells and that samples with similar ELISpot results could therefore have different ELISA results. In animal models this observation was also associated with different disease outcomes. ${ }^{29}$ Further studies are therefore needed to assess the clinical relevance of these findings.

Regression analyses showed that individuals with high $M$ tuberculosis exposure were more likely to have a positive IGRA. This is consistent with a recent household contact study in Nigeria; children exposed to a smear-positive index case were more likely to be Quantiferon-in-tube positive than those with a smear-negative index case. ${ }^{30}$ Our data suggest that the use of an exposure gradient to $M$ tuberculosis in high burden settings such as South Africa is feasible. Exposure gradients need to be further validated and refined in tuberculosis endemic settings to elucidate differences between risk factors for household and other sources of transmission. Our composite contact score allowed for multiple factors including smear status, duration and proximity of exposure. The enhanced performance of the $\mathrm{T}$ -

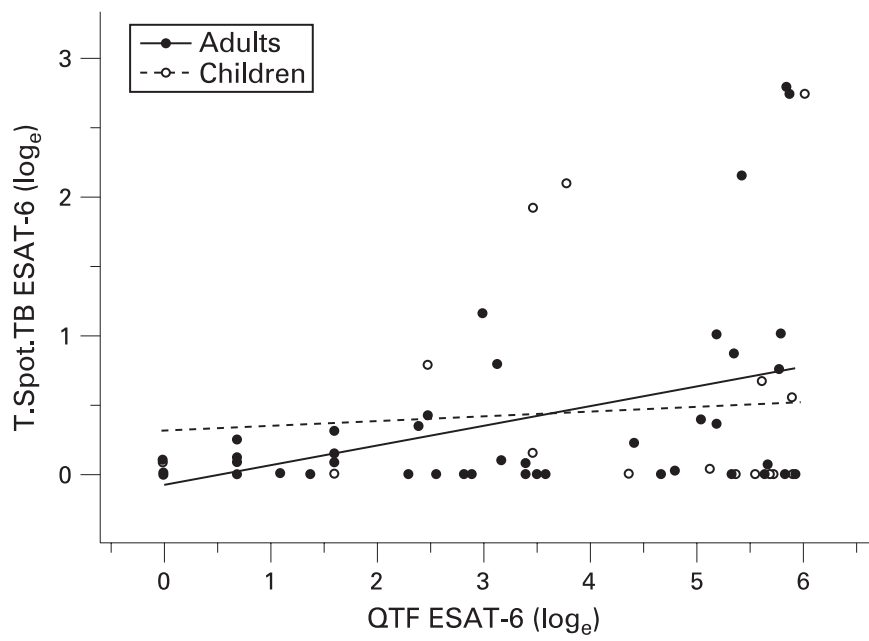

Figure 3 Correlation between interferon $\gamma$ (IFN $\gamma$ ) early secretory antigenic target (ESAT)- 6 responses measured by QuantiFERON TB Gold (OTF) and T-SPOT.TB in adults and children. Vertical axis: T-SPOT.TB ESAT-6 IFN $\gamma$ responses (spot count per 250000 peripheral blood mononuclear cells); horizontal axis: OTF IFN $\gamma$ ESAT-6 response (IU/ml); both reflected on the natural log scale. Spearman rank correlation coefficient for adults $r=0.34$ and for children $r=-0.06 ; p=0.135$ for comparison of coefficients between groups. 


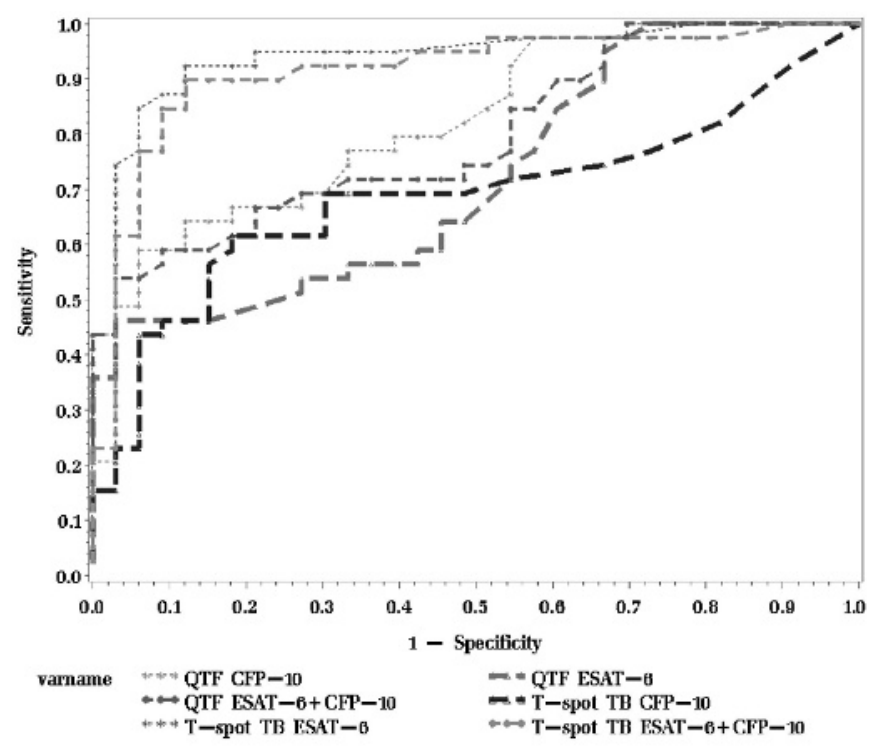

$\begin{array}{lc} & \text { Area } \\ \text { QTF ESAT-6 } & 0.721 \\ \text { QTF CFP-10 } & 0.825 \\ \text { QTF ESAT-6 + CFP-10 } & 0.796 \\ \text { T SPOT.TB ESAT-6 (Panel A) } & 0.932 \\ \text { T SPOT.TB CFP-10 (Panel B) } & 0.687 \\ \text { T SPOT.TB ESAT-6 + CFP-10 (Panel A + Panel B) } & 0.914\end{array}$

Figure 4 Receiver operating curves (ROC) for individual and mean combined early secretory antigenic target (ESAT)-6 and culture filtrate protein $10 \mathrm{kD}$ (CFP-10) interferon $\gamma$ (IFN $\gamma$ ) responses for T-SPOT.TB and Quantiferon-Gold (QTF) in adults and children in relation to high $M$ tuberculosis exposure (contact score $\geqslant 4$ ). Individual antigen responses for T-SPOT.TB: panel A; ESAT-6, and panel B; CFP-10 spot count, and IFN $\gamma$ OTF antigen response (IU/ml) to ESAT-6 and CFP10.

SPOT.TB compared with the QTF as assessed through exploratory ROC analysis in relation to $M$ tuberculosis exposure also requires further validation.

The consideration of age-related differences between IGRA test agreement in adults and children is important as test responses may be dependent on both age and recent degree of $M$ tuberculosis exposure. Although our study had limited power to measure the impact of age on the relationship between $M$ tuberculosis exposure and IGRAs, our results suggest that the odds of having a positive IGRA result increased when controlling for age. Chee et al have demonstrated different age-related responses to the T-SPOT.TB for the two antigens used in adults with $M$ tuberculosis infection. ${ }^{31}$ Our results also indicate a potential age-related correlation between antigen responses. Potential mechanisms for these observations may include age-dependent immune 1 maturation.

This study is limited by its cross-sectional nature and its small sample size, as reflected in wide confidence intervals around effect estimates despite statistically significant results for main outcomes. Other limitations include the age distribution and lack of serial measures to assess discordant results and test reproducibility. Since this was a household contact study, we did not assess individuals without documented $M$ tuberculosis exposure; future studies should incorporate the use of such control populations and should ideally include serial measurements of both exposure and infection. We do not report on findings in HIV-infected patients. ELISPOT-based IGRAs have been shown to be sensitive measures of $M$ tuberculosis infection in HIV-infected individuals in endemic settings and also in those with decreased CD4+ T cell counts. ${ }^{20}{ }^{32} 33$ The QTF was not completed in all children owing to inadequate blood volumes. Finally, although the QTF used in this study is still in use, a newer in-tube version which includes TB 7.7 as an additional antigen is currently being marketed and differences between responses measured by different generations of QTF test may be considerable. ${ }^{34}$ An important potential distinction which we have retained by using the OTF in the present study is the ability to distinguish between different antigen responses; this has been lost using the newer generation in-tube QTF which combines all three antigens.

In summary, we found that the T-SPOT.TB, OTF and TST were highly discordant in adults and children in recent household contacts. The T-SPOT.TB correlated better with high exposure levels in young child contacts. Larger communitybased longitudinal studies with serial assessment of discordant results and accurate long-term outcome measures are essential to assess the predictive value of IGRAs for infection and disease in HIV-uninfected and infected children and adults. Further studies should also assess age-related individual IGRA antigen responses and the use of more comprehensive exposure gradients as a surrogate measure of infection.

Acknowledgements: The authors thank the study nurses, the TB clinic staff in Ravensmead and Uitsig, the patients and their families for their kind cooperation, the Cape Town City TB directorate and Dr Ivan Toms for permission to conduct this study, and Professor Mark Doherty, Statens Serum Institute, Denmark for helpful advice regarding technical aspects of the assays used in this study.

Funding: Supported by the South African National Research Foundation NRF Thuthuka TTK2006051500016 (ACH), NIH IK23-HD40982 (AMM) and the Bill and Melinda Gates Foundation through Grand Challenges in Global Health Grant 37772 (NNC, KS, GB, GW) and the Norwegian Centre or Cooperation in Higher Education (ACH, GW).

\section{Competing interests: None.}

Ethics approval: Informed consent was obtained for participation and HIV testing. Institutional approval was obtained from Stellenbosch University.

\section{REFERENCES}

1. Dolin PJ, Raviglione MC, Kochi A. Global tuberculosis incidence and mortality during 1990-2000. Bull WHO 1994;72:213-20.

2. Nelson LJ, Schneider E, Wells CD, et al. Epidemiology of childhood tuberculosis in the United States, 1993-2001: the need for continued vigilance. Pediatrics 2004;114:333-41.

3. Marais BJ, Gie RP, Schaaf HS, et al. The natural history of childhood intra-thoracic tuberculosis: a critical review of literature from the pre-chemotherapy era. Int J Tuberc Lung Dis 2004;8:392-402.

4. International Union against Tuberculosis and Lung Disease. Epidemiologic basis of tuberculosis control. 1st ed. Paris: International Union against Tuberculosis and Lung Disease, 1999

5. Schaaf HS, Michaelis IA, Richardson M, et al. Adult-to-child transmission of tuberculosis: household or community contact? Int J Tuberc Lung Dis 2003;7:426-31.

6. Wilkinson KA, Kon OM, Newton SM, et al. Effect of treatment of latent tuberculosis infection on the T cell response to Mycobacterium tuberculosis antigens. J Infect Dis 2006;193:354-9.

7. Menzies D, Pai M, Comstock G. Meta-analysis: new tests for the diagnosis of latent tuberculosis infection: areas of uncertainty and recommendations for research. Ann Intern Med 2007;:146:340-54.

8. Anon. Tuberculosis. In: Pickering LK, ed. Red Book: 2006 Report of the Committee on Infectious Diseases. 27th ed. Elk Grove Village: American Academy of Pediatrics, 2006:678-98.

9. Jeffries DJ, Hill PC, Fox A, et al. Identifying ELISPOT and skin test cut-offs for diagnosis of Mycobacterium tuberculosis infection in the Gambia. Int J Tuberc Lung Dis 2006;10:192-8.

10. Jachuck SJ, Bound $C L$, Price $P$. The importance of tuberculin retesting in the adult community at risk. J $R$ Coll Gen Pract 1984;34:210-2.

11. Hill PC, Brookes RH, Fox A, et al. Longitudinal assessment of an ELISPOT test for Mycobacterium tuberculosis infection. PLoS Med 2007:4:e192.

12. Ewer K, Deeks J, Alvarez L, et al. Comparison of T-cell-based assay with tuberculin skin test for diagnosis of Mycobacterium tuberculosis infection in a school tuberculosis outbreak. Lancet 2003;361:1168-73.

13. Hill PC, Brookes RH, Fox A, et al. Large-scale evaluation of enzyme-linked immunospot assay and skin test for diagnosis of Mycobacterium tuberculosis infection against a gradient of exposure in the Gambia. Clin Infect Dis 2004;38:966-73. 
14. Richeldi L, Ewer K, Losi M, et al. T cell-based tracking of multidrug resistant tuberculosis infection after brief exposure. Am J Respir Crit Care Med 2004:170:288-95.

15. Marais BJ, Hesseling AC, Gie RP, et al. T he burden of childhood tuberculosis and the accuracy of community-based surveillance data. Int J Tuberc Lung Dis 2006:10:259-63.

16. Den Boon S, Verver S, Marais BJ, et al. Association of passive smoking and Mycobacterium tuberculosis infection in children. Pediatrics 2007;119:724-39.

17. van der Spuy GD, Warren RM, Richardson M, et al. Use of genetic distance as a measure of ongoing transmission of Mycobacterium tuberculosis. J Clin Microbiol 2003:41:5640-4.

18. Shams H, Weis SE, Klucar $\mathrm{P}$, et al. Enzyme-linked immunospot and tuberculin skin testing to detect latent tuberculosis infection. Am J Respir Crit Care Med 2005;172:1161-8.

19. Soysal A, Torun T, Efe $S$, et al. Evaluation of cut-off values of interferon-gammabased assays in the diagnosis of $\mathrm{M}$ tuberculosis infection. Int J Tuberc Lung Dis 2008:12:50-6.

20. Mandalakas AM, Hesseling AC, Chegou NN, et al. High level of discordant IGRA results in HIV-infected adult and children. Int J Tuberc Lung Dis 2008;12:417-23.

21. Rangaka MX, Wilkinson KA, Seldon R, et al. Effect of HIV-1 infection on T-cell-based and skin test detection of tuberculosis infection. Am J Respir Crit Care Med 2007:175:514-20.

22. Connell TG, Curtis N, Ranganathan SC, et al. Performance of a whole blood interferon gamma assay for detecting latent infection with Mycobacterium tuberculosis in children. Thorax 2006;61:616-20.

23. Tsiouris SJ, Austin J, Toro P, et al. Results of a tuberculosis-specific IFN-gamma assay in children at high risk for tuberculosis infection. Int J Tuberc Lung Dis 2006;10:939-41.

24. Dogra S, Narang P, Mendiratta DK, et al. Comparison of a whole blood interferon-gamma assay with tuberculin skin testing for the detection of tuberculosis infection in hospitalized children in rural India. J Infect 2007:54:267-76.
25. Hill PC, Jeffries DJ, Brookes RH, et al. Using ELISPOT to expose false positive skin test conversion in tuberculosis contacts. PLOS ONE 2007:2:e183

26. Lienhardt C, Sillah J, Fielding K, et al. Risk factors for tuberculosis infection in children in contact with infectious tuberculosis cases in the Gambia, West Africa. Pediatrics 2003;111(5 Pt 1):e608-14.

27. Adetifa IM, Lugos MD, Hammond $A$, et al. Comparison of two interferon gamma release assays in the diagnosis of Mycobacterium tuberculosis infection and disease in the Gambia. BMC Infect Dis 2007; 7:122.

28. Leyten EM, Arend SM, Prins C, et al. Discrepancy between Mycobacterium tuberculosis-specific gamma interferon release assays using short and prolonged in vitro incubation. Clin Vaccine Immunol 2007:880-5

29. Bennekov T, Dietrich J, Rosenkrands I, et al. Alteration of epitope recognition pattern in Ag85B and ESAT-6 has a profound influence on vaccine-induced protection against Mycobacterium tuberculosis. Eur J Immunol 2006;36:3346-55.

30. Nakaoka H, Lawson L, Squire SB, et al. Risk for tuberculosis among children. Emerg Infect Dis 2006:12:1383-8.

31. Chee CB, KhinMar KW, Gan SH, et al. Latent tuberculosis infection treatment and Tcell responses to Mycobacterium tuberculosis-specific antigens. Am J Respir Crit Care Med 2007:175:282-7.

32. Chapman AL, Munkanta M, Wilkinson KA, et al. Rapid detection of active and latent tuberculosis infection in HIV-positive individuals by enumeration of Mycobacterium tuberculosis-specific T cells. AIDS 2002;16:2285-93.

33. Liebeschuetz S, Bamber S, Ewer K, et al. Diagnosis of tuberculosis in South African children with a T-cell-based assay: a prospective cohort study. Lancet 2004;364:2196-203.

34. Mahomed H, Hughes EJ, Hawkridge T, et al. Comparison of Mantoux skin test with three generations of a whole blood IFN-gamma assay for tuberculosis infection. Int J Tuberc Lung Dis 2006:10:310-6.

\section{BMJ Masterclasses}

BMJ Masterclasses are educational meetings designed specifically to meet the learning needs of doctors. They help doctors keep up to date with the latest evidence and recent guidelines in major clinical areas, enabling them to use the latest evidence to make better decisions. The latest evidence, recent guidelines and best practice are delivered in an interactive and informative manner by leading experts. The speakers are specifically chosen as highly-skilled communicators who can authoritatively enthuse the audience and interpret the latest research and guidelines into practical tips for busy doctors. BMJ Masterclasses have proved a huge hit with clinicians, with many saying they have influenced their clinical practice.

http://masterclasses.bmj.com/

BMJ

masterclasses 